\title{
Statistics of VHE $\gamma$-rays in temporal association with radio giant pulses from the Crab pulsar
}

\author{
MAGIC Collaboration: M. L. Ahnen ${ }^{1}$, S. Ansoldi ${ }^{2,3}$, L. A. Antonelli ${ }^{4}$, C. Arcaro ${ }^{5}$, A. Babić ${ }^{6}$, B. Banerjee ${ }^{7}$,
} P. Bangale ${ }^{8}$, U. Barres de Almeida ${ }^{8,9}$, J. A. Barrio ${ }^{10}$, J. Becerra González ${ }^{11}$, W. Bednarek ${ }^{12}$, E. Bernardini ${ }^{13,14}$, A. Berti ${ }^{2}, 15$, W. Bhattacharyya ${ }^{13}$, B. Biasuzzi ${ }^{2}$, A. Biland ${ }^{1}$, O. Blanch ${ }^{16}$, G. Bonnoli ${ }^{17}$, R. Carosi ${ }^{17}$, A. Carosi ${ }^{4}$, A. Chatterjee ${ }^{7}$, S. M. Colak ${ }^{16}$, P. Colin ${ }^{8}$, E. Colombo ${ }^{11}$, J. L. Contreras ${ }^{10}$, J. Cortina ${ }^{16}$, S. Covino ${ }^{4}$, P. Cumani ${ }^{16}$,

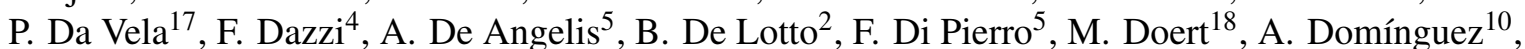

D. Dominis Prester ${ }^{6}$, D. Dorner ${ }^{19}$, M. Doro ${ }^{5}$, S. Einecke ${ }^{18}$, D. Eisenacher Glawion ${ }^{19}$, D. Elsaesser ${ }^{18}$, M. Engelkemeier ${ }^{18}$, V. Fallah Ramazani ${ }^{20}$, A. Fernández-Barral ${ }^{16}$, D. Fidalgo ${ }^{10}$, M. V. Fonseca ${ }^{10}$, L. Font $^{21}$, C. Fruck ${ }^{8}$, D. Galindo ${ }^{22}$, R. J. García López ${ }^{11}$, M. Garczarczyk ${ }^{13}$, M. Gaug ${ }^{21}$, P. Giammaria $^{4}$, N. Godinović ${ }^{6}$,

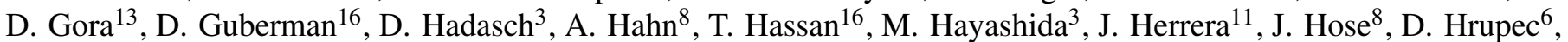
K. Ishio $^{8}$, Y. Konno ${ }^{3}$, H. Kubo ${ }^{3}$, J. Kushida ${ }^{3}$, D. Kuveždić ${ }^{6}$, D. Lelas ${ }^{6}$, N. Lewandowska ${ }^{19,23 \star}$, E. Lindfors ${ }^{20}$, S. Lombardi ${ }^{4}$, F. Longo ${ }^{2,15}$, M. López ${ }^{10}$, C. Maggio ${ }^{21}$, P. Majumdar ${ }^{7}$, M. Makariev ${ }^{24}$, G. Maneva ${ }^{24}$, M. Manganaro ${ }^{6}$, K. Mannheim ${ }^{19}$, L. Maraschi ${ }^{4}$, M. Mariotti ${ }^{5}$, M. Martínez ${ }^{16}$, D. Mazin ${ }^{8,3}$, U. Menzel ${ }^{8}$, M. Minev ${ }^{24}$, J. M. Miranda ${ }^{17}$,

R. Mirzoyan ${ }^{8}$, A. Moralejo ${ }^{16}$, V. Moreno ${ }^{21}$, E. Moretti ${ }^{8}$, T. Nagayoshi ${ }^{3}$, V. Neustroev ${ }^{20}$, A. Niedzwiecki ${ }^{12}$, M. Nievas Rosillo ${ }^{10}$, C. Nigro ${ }^{13}$, K. Nilsson ${ }^{20}$, D. Ninci ${ }^{16}$, K. Nishijima ${ }^{3}$, K. Noda ${ }^{16}$, L. Nogués ${ }^{16}$, S. Paiano ${ }^{5}$, J. Palacio ${ }^{16}$, D. Paneque ${ }^{8}$, R. Paoletti ${ }^{17}$, J. M. Paredes ${ }^{22}$, G. Pedaletti ${ }^{13}$, M. Peresano ${ }^{2}$, L. Perri ${ }^{4}$, M. Persic ${ }^{2,25}$, P. G. Prada Moroni ${ }^{26}$, E. Prandini ${ }^{5}$, I. Puljak ${ }^{6}$, J. R. Garcia ${ }^{8}$, I. Reichardt ${ }^{5}$, W. Rhode ${ }^{18}$, M. Ribó ${ }^{22}$, J. Rico ${ }^{16}$, C. Righi ${ }^{4}$, A. Rugliancich ${ }^{17}$, T. Saito ${ }^{3, \star}$, K. Satalecka ${ }^{13}$, S. Schroeder ${ }^{18}$, T. Schweizer ${ }^{8}$, S. N. Shore ${ }^{26}$, J. Sitarek ${ }^{12}$, I. Šnidarić6 ${ }^{\text {, D. Sobczynska }}{ }^{12}$, A. Stamerra ${ }^{4}$, M. Strzys ${ }^{8}$, T. Surić6 ${ }^{6}$ L. Takalo ${ }^{20}$, F. Tavecchio ${ }^{4}$, P. Temnikov ${ }^{24}$,

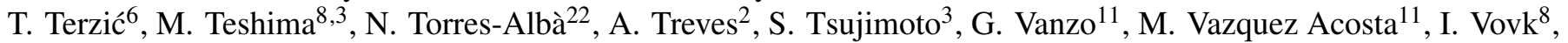
J. E. Ward ${ }^{16}$, M. Will ${ }^{8}$, and D. Zarić6; Radio Collaborators: R. Smits ${ }^{27}$

(Affiliations can be found after the references)

Received 1 June 2018 / Accepted 30 October 2019

\section{ABSTRACT}

Aims. The aim of this study is to search for evidence of a common emission engine between radio giant pulses (GPs) and very-high-energy (VHE, $E>100 \mathrm{GeV}) \gamma$-rays from the Crab pulsar.

Methods. We performed $16 \mathrm{~h}$ of simultaneous observations of the Crab pulsar at $1.4 \mathrm{GHz}$ with the Effelsberg radio telescope and the Westerbork Synthesis Radio Telescope (WSRT), and at energies above $60 \mathrm{GeV}$ we used the Major Atmospheric Gamma-ray Imaging Cherenkov (MAGIC) telescopes. We searched for a statistical correlation between the radio and VHE $\gamma$-ray emission with search windows of different lengths and different time lags to the arrival times of a radio GP. A dedicated search for an enhancement in the number of VHE $\gamma$-rays correlated with the occurrence of radio GPs was carried out separately for the P1 and P2 phase ranges, respectively.

Results. In the radio data sample, 99444 radio GPs were detected. We find no significant correlation between the GPs and VHE photons in any of the search windows. Depending on phase cuts and the chosen search windows, we find upper limits at a 95\% confidence level on an increase in VHE $\gamma$-ray events correlated with radio GPs between $7 \%$ and $61 \%$ of the average Crab pulsar VHE flux for the P1 and P2 phase ranges, respectively. This puts upper limits on the flux increase during a radio GP between $12 \%$ and $2900 \%$ of the pulsed VHE flux, depending on the search window duration and phase cuts. This is the most stringent upper limit on a correlation between $\gamma$-ray emission and radio GPs reported so far.

Key words. pulsars: individual: Crab pulsar - gamma rays: stars - radio continuum: stars - radiation mechanisms: non-thermal

\section{Introduction}

Since the discovery of the first pulsar (Hewish et al. 1968), more than 2500 of these objects have been found (Manchester et al. 2005). A large variety of emission properties have been observed

\footnotetext{
* Corresponding authors: N. Lewandowska (e-mail: natalia.lewandowska@mail.wvu.edu), T. Saito (e-mail: tsaito@icrr.u-tokyo.ac.jp).
}

in these objects, leading to the designation of diverse populations in the literature (see review by Harding 2013). Some pulsars are observed only at certain wavelengths, while others can be observed throughout large parts of the electromagnetic spectrum. The Crab pulsar has been observed so far from about $10^{-8} \mathrm{eV}\left(20 \mathrm{MHz}\right.$, Ellingson et al. 2013) up to $1.5 \times 10^{12} \mathrm{eV}$ (Ansoldi et al. 2016). The approximate alignment of its pulsed emission across the electromagnetic spectrum (time delays were 
reported by Oosterbroek et al. 2008) suggests a common engine for its broadband pulsed emission. The Crab pulsar is, therefore, a suitable object to test various emission theories, explaining the generation of its multi-wavelength emission.

The average pulse profile of the Crab pulsar changes with frequency, showing up to seven different components (Moffett \& Hankins 1996; Hankins et al. 2015). In the radio band, below $5 \mathrm{GHz}$, it consists of the main pulse (MP, at a rotation phase from $\sim-0.01$ to $\sim 0.01$ ), the low frequency interpulse (LFIP, from $\sim 0.39$ to $\sim 0.42$ in phase), the precursor (PC, from $\sim-0.07$ to $\sim-0.02$ in phase, only below $0.6 \mathrm{GHz}$ ) and the low frequency component (LFC, from $\sim-0.14$ to $\sim-0.07$ in phase, only between 0.6 and $4.2 \mathrm{GHz}$ ). At above $5 \mathrm{GHz}$, the MP vanishes and an additional interpulse component known as high frequency interpulse (HFIP) occurs, which is shifted by about 0.02 with regard to the LFIP and located at $\sim 0.36$ to $\sim 0.42$ in phase. In addition, two components known as high frequency components (HFC1 at $\sim 0.53$ to $\sim 0.67$ in phase, HFC2 at $\sim 0.68$ to $\sim 0.81$ in phase) appear (Moffett \& Hankins 1996; Hankins et al. 2015). The names of all of the components and corresponding phases are summarized in Table 1.

Extensive single pulse studies of the Crab pulsar below and above about $5 \mathrm{GHz}$ show even more complex features (Hankins et al. 2016). While MP and LFIP single pulses consist of several microsecond long bursts, which can be resolved into single pulses of a duration of nanoseconds with continuous spectra across the observing band, HFIP single pulses consist of one burst of emission of a duration of several microseconds with non-uniform spectra in the form of proportionally spaced emission bands (Hankins et al. 2016). No single pulses of nanosecond duration were detected in the case of HFIP single pulses. The observed differences, therefore, suggest similar emission physics for MP and LFIP single pulses and different ones for HFIP single pulses (Hankins et al. 2016).

Single pulses whose flux density is more than ten times higher than the mean are called giant pulses (GPs, Karuppusamy et al. 2010). The pulse widths of radio GPs from the Crab pulsar are in the microseconds to nanoseconds range (Hankins et al. 2003) and their intensity distributions can be described by a powerlaw (Argyle \& Gower 1972). The shortest widths observed so far have been reported to be less than $0.4 \mathrm{~ns}$, resulting in a brightness temperature of about $10^{41} \mathrm{~K}$ (Hankins \& Eilek 2007). The high brightness temperatures imply a coherent emission mechanism (Hankins et al. 2009). Strong and frequent radio GPs are observed mainly at the phase ranges of MP, LFIP, and HFIP (Jessner et al. 2010; Hankins et al. 2012). Such a complex evolution of the average profile at radio wavelengths has never been observed in any other pulsar so far.

In the $\gamma$-ray band, the average pulse profile is smoother and broader than at radio frequencies (Kuiper et al. 2001; Abdo et al. 2010; VERITAS Collaboration 2011; Aleksić et al. 2012). Rotation phases between -0.01 to $\sim 0.1$ are often called the "P1", the ones between 0.3 and 0.5 are the "P2", and the ones between $\sim 0.1$ and 0.3 are known as the "Bridge" (Fierro et al. 1998; Aleksic et al. 2014). It is important to note that the MP is included in the P1 range, while LFIP and HFIP are in the P2 range as shown in Table 1.

Because of the above mentioned high energy density of GPs in small volumes, a correlation between radio GPs and emission at higher energy bands can be hypothesized (e.g., Eilek \& Hankins 2016). One process that could facilitate the required energy release on short spatial and temporal scales is magnetic reconnection in the current sheet outside the light cylinder. In this process, kinetic instabilities break the frozen-in condition of ideal magnetohydrodynamics (MHD; Contopoulos \& Kalapotharakos 2010; Tchekhovskoy et al. 2013), which holds at large ${ }^{1}$ scales and converts magnetic energy into kinetic energy of high energy particles. Both particle-in-cell simulations (Spitkovsky 2006; Cerutti et al. 2012) and analytical descriptions (Contopoulos et al. 1999; Contopoulos 2007) of the pulsar magnetosphere confirmed the existence of current sheets and demonstrated the important role that the magnetic reconnection mechanism can play (Uzdensky \& Spitkovsky 2014). Each stochastically occurring reconnection event would produce radio and high energy emission from synchrotron emission of the energetic particles, for example. Even if a comprehensive theoretical framework does not exist yet, the possibility of finding such a correlation between radio and $\gamma$-rays triggered different observations in the $\gamma$-ray band.

The Crab pulsar has been also extensively studied in the very-high-energy (VHE) $\gamma$-ray range. Imaging Air Cherenkov Telescopes (IACTs) like MAGIC (Major Atmospheric Gammaray Imaging Cherenkov telescopes) and VERITAS (Very Energetic Radiation Imaging Telescope Array System) revealed that the $\mathrm{P} 2$ component is dominant above $50 \mathrm{GeV}$ up to $1.5 \mathrm{TeV}$, while the P1 component has been measured up to $600 \mathrm{GeV}$ (Aliu et al. 2008; VERITAS Collaboration 2011; Aleksić et al. 2012; Ansoldi et al. 2016). The bridge emission is significantly detected only up to $\sim 150 \mathrm{GeV}$ (Aleksić et al. 2014). Any pulsed emission above $25 \mathrm{GeV}$ cannot be explained by the conventional polar-cap pulsar models (Ruderman \& Sutherland 1975; Daugherty \& Harding 1982; Baring 2004) and challenges the slot-gap scenario (Harding et al. 2008), while outer-gap models in which $\gamma$-rays are produced by curvature radiation of electrons accelerated in the magnetosphere (Hirotani 2008; Tang et al. 2008) are favored.

In the present work, we explore the association between radio GP and VHE $(E>100 \mathrm{GeV}) \gamma$-rays. Separate analyses have been conducted in order to search for evidence of common emission between GPs and VHE $\gamma$-rays for each of the two $\gamma$-ray peaks P1 and P2 (and corresponding radio phases MP and LFIP respectively). From now on, adopting the notation of Aleksić et al. (2014), we refer to P1 GPs and P2 GPs to indicate GPs falling inside the VHE $\gamma$-rays phase range $[-0.01$ to 0.02$]$ and [0.37-0.42] respectively: due to the radio frequency considered in the present work $(1.4 \mathrm{GHz})$, this translates to MP GPs and LFIP GPs.

Given that the origin of GPs is not known, it is certainly interesting to search for a correlation between radio GPs and VHE pulsed photons, although there is currently no theoretical approach which describes their correlation. In fact, several searches for multi-wavelength counterparts of radio GPs and optical photons were reported with $7.8 \sigma$ (Shearer et al. 2003) and 7.2 $\sigma$ (Strader et al. 2013) significance for MP GPs and $1.75 \sigma$ (Shearer et al. 2003) and 3.5 $\sigma$ (Strader et al. 2013) for LFIP GPs. This result implies the existence of an additional incoherent emission mechanism associated with radio GPs from the Crab pulsar. Similar studies were carried out in the X-ray band, finding no correlation (Bilous et al. 2012; Mikami et al. 2013, 2014; Aharonian 2018).

Past searches for a correlation between radio GPs and $\gamma$-rays from the Crab pulsar provided no positive results either (Argyle et al. 1974; Lundgren et al. 1995; Bilous et al. 2011; Mickaliger et al. 2012). The only other recent study for which data from an IACT was used was carried out by VERITAS (Aliu et al. 2012), who searched for a correlation between radio

larger than the kinetic length scales in the plasma. 
M. L. Ahnen et al.: Statistics of VHE $\gamma$-rays in temporal association with radio giant pulses from the Crab pulsar

Table 1. Rotational phase ranges, frequency ranges of occurrence, and nomenclature of average emission components of the Crab pulsar.

\begin{tabular}{|c|c|c|c|c|}
\hline \multirow[b]{2}{*}{ Name } & \multicolumn{2}{|c|}{ Radio } & \multicolumn{2}{|r|}{$\gamma$-ray } \\
\hline & $\begin{array}{c}\text { Phase range }^{(1)} \\
\text { [Periods] }\end{array}$ & $\begin{array}{c}\text { Frequency } \\
\text { [GHz] }\end{array}$ & Name & $\begin{array}{c}\text { Phase range }^{(2)} \\
\text { [Periods] }\end{array}$ \\
\hline LFC & $\sim-0.14$ to $\sim-0.07$ & $0.6-4.2$ & & \\
\hline $\mathrm{PC}$ & $\sim-0.07$ to $\sim-0.02$ & $0.3-0.6$ & P1 & $\sim-0.01$ to $\sim 0.02$ \\
\hline \multirow[t]{2}{*}{ MP } & $\sim-0.01$ to $\sim 0.01$ & $0.3-4.9$ & & \\
\hline & & & Bridge & $\sim 0.02$ to $\sim 0.37$ \\
\hline HFIP & $\sim 0.36$ to $\sim 0.42$ & $4.2-28.4$ & P2 & $\sim 0.37$ to $\sim 0.42$ \\
\hline LFIP & $\sim 0.39$ to $\sim 0.42$ & $0.3-3.5$ & & \\
\hline HFC1 & $\sim 0.53$ to $\sim 0.67$ & $1.4-28.0$ & Off-pulse & $\sim 0.52$ to $\sim 0.87$ \\
\hline HFC2 & $\sim 0.68$ to $\sim 0.81$ & $1.4-28.0$ & & \\
\hline
\end{tabular}

Notes. ${ }^{(1)}$ Radio phase ranges and frequency values are taken from Hankins et al. (2015). ${ }^{(2)} \gamma$-ray phase ranges are taken from Aleksić et al. (2014).

GPs at $8.9 \mathrm{GHz}$ and VHE $\gamma$-rays with energies higher than $150 \mathrm{GeV}$. With a total overlap of $11.6 \mathrm{~h}$, they reported upper limits of five to ten times the average Crab pulsar VHE flux on the flux measured simultaneously with P2 GPs and of two to three times the average VHE flux on time scales of about eight seconds around P2 GPs. The present study focuses on the search for a correlation between radio GPs from the Crab pulsar and its VHE $\gamma$-ray emission. The differences with respect to the study carried out by the VERITAS Collaboration in Aliu et al. (2012) are the following: firstly, the corresponding radio data presented here were taken at a center frequency of about $1.4 \mathrm{GHz}$, whereas radio observations described in Aliu et al. (2012) were carried out at $8.9 \mathrm{GHz}$. Based on the results by Hankins et al. (2016) we are addressing a different population of radio GPs. Secondly, the $\gamma$-ray observations reported here were carried out at energies above $60 \mathrm{GeV}$, where the $\mathrm{P} 1$ emission is pronounced, while in Aliu et al. (2012) the energy threshold was above $150 \mathrm{GeV}$, where the $P 1$ emission is much fainter (Aleksić et al. 2012). The lower energy threshold of MAGIC $\left(E_{\mathrm{thr}} \sim 60 \mathrm{GeV}\right)$ in comparison with the energy threshold of VERITAS $\left(E_{\mathrm{thr}} \sim 150 \mathrm{GeV}\right)$ allows a more comprehensive analysis of the correlation between VHE $\gamma$-rays and the P1 GPs. Thirdly, the amount of simultaneous observations between VHE $\gamma$-rays and radio is larger in the present study (16h vs. $11.6 \mathrm{~h}$ ), corresponding to the currently largest sample of simultaneous VHE $\gamma$-rays and radio GP data taken with an IACT.

The paper is organized as follows: the observations and data analysis are described in Sect. 2. The construction of the Monte Carlo (MC) simulations is described together with the correlation study in Sect. 3. The results are discussed in Sect. 4 and a summary can be found in Sect. 5. The Appendix A carries a detailed explanation of the MC simulations developed specifically for this study.

\section{Observations and data reduction}

\subsection{Radio observations}

The radio observations were carried out with the Effelsberg radio telescope and the Westerbork Synthesis Radio Telescope (WSRT) at a frequency of about $1.4 \mathrm{GHz}$. The two facilities scheduled complementary observations in order to exclude overlaps in the recorded data sample. Observations of the Crab pulsar with the Effelsberg radio telescope were carried out in baseband mode with the $\mathrm{P} 217 \mathrm{~mm}$ and $\mathrm{P} 200 \mathrm{~mm}$ prime focus receivers and the PSRIX pulsar backend (Lazarus et al. 2016). The Crab pulsar observations taken with the WSRT were carried out with 13 out of 14 available antennas, their Multi-frequency Front End Receivers (MFFEs, Casse et al. 1982; Tan et al. 1991), and the PuMa II pulsar backend (Karuppusamy et al. 2008).

All radio data sets were coherently dedispersed (Hankins \& Rickett 1975) during an off-line reduction process. For this part of the reduction the digital library DSPSR (van Straten \& Bailes 2011) was used. After the dedispersion procedure, the resulting data sets were phase folded with ephemeris files obtained from the Jodrell Bank Observatory (Lyne et al. 1993). To ensure absolute alignment between the radio and $\gamma$-rays pulses, an ephemeris which covered the observing days was created and used instead of the monthly released one. To extract the brightest single pulses, an additional data selection, based on the standard deviation of the signal in the OFF-pulse radio emission regions, was introduced in the dedispersed data sets. With this technique, a total number of 99444 GPs was extracted from the radio data. A summary of all the radio observations performed for this study is given in Table 2 and a corresponding phase diagram of an observation taken with the Effelsberg telescope and the WSRT is shown in Fig. 1.

\section{2. $\gamma$-ray observations}

VHE $\gamma$-ray observations were carried out with the MAGIC telescopes between December 2012 and February 2013 (simultaneously with observations either with the Effelsberg radio telescope, or the WSRT). They were taken at zenith angles of less than $30^{\circ}$ to achieve the lowest possible energy threshold, and with both telescopes in Wobble observation mode (Fomin et al. 1994). The reduction of the resulting data was carried out according to the standard analysis pipeline using the MAGIC Analysis and Reconstruction Software (MARS, Zanin et al. 2013).

To efficiently suppress the hadronic background without losing a large fraction of air showers induced by VHE photons from the Crab pulsar, energy dependent cuts in Hadroness (a test statistic for discrimination between a $\gamma$-ray or a hadron induced shower) and $\theta^{2}$ (the squared angular distance between the expected source position and the reconstructed one) parameters were performed (details in Aleksić et al. 2016). They were optimized on an independent data sample of $46 \mathrm{~h}$ of observations, taken at zenith angles of less than $30^{\circ}$, same as the main data set used in the present work. For an energy range spanning from $5 \mathrm{GeV}$ to $50 \mathrm{TeV}, 30$ logarithmic energy bins were defined. In each energy bin the Hadroness and $\theta^{2}$ parameters 
Table 2. Summary of radio and VHE $\gamma$-ray observations.

\begin{tabular}{lrrrrrrr}
\hline \hline $\begin{array}{l}\text { Epoch } \\
\text { [YYYY-MM-DD] }\end{array}$ & $\begin{array}{r}v \\
{[\mathrm{MHz}]}\end{array}$ & $\begin{array}{r}\text { BW } \\
{[\mathrm{MHz}]}\end{array}$ & Facility & $N_{\mathrm{GP}}$ & $\begin{array}{r}T_{\text {radio }} \\
{[\mathrm{h}]}\end{array}$ & $\begin{array}{r}T_{\text {MAGIC }} \\
{[\mathrm{h}]}\end{array}$ & $\begin{array}{r}T_{\text {overlap }} \\
{[\mathrm{h}]}\end{array}$ \\
\hline $2012-12-07$ & 1347.5 & 200 & Eff & 1687 & 1.8 & 2.9 & 0.8 \\
$2012-12-10$ & 1380.0 & 160 & WSRT & 15456 & 2.0 & 2.0 & 1.9 \\
$2012-12-17$ & 1347.5 & 200 & Eff & 3429 & 2.3 & 1.7 & 1.6 \\
$2013-01-08$ & 1380.0 & 160 & WSRT & 5058 & 0.4 & 1.8 & 0.3 \\
$2013-01-09$ & 1372.5 & 200 & Eff & 3525 & 1.7 & 1.9 & 1.2 \\
$2013-01-10$ & 1380.0 & 160 & WSRT & 24274 & 2.0 & 1.8 & 1.5 \\
$2013-01-12$ & 1347.5 & 200 & Eff & 6445 & 2.8 & 1.8 & 1.4 \\
$2013-01-31$ & 1347.5 & 200 & Eff & 1688 & 1.5 & 2.0 & .0 .7 \\
$2013-02-02$ & 1380.0 & 160 & WSRT & 7118 & 0.9 & 2.0 & 0.9 \\
$2013-02-03$ & 1380.0 & 160 & WSRT & 18392 & 2.0 & 1.8 & 1.7 \\
$2013-02-06$ & 1347.5 & 200 & Eff & 4470 & 2.2 & 1.3 & 0.1 \\
$2013-02-07$ & 1410.0 & 75 & Eff & 696 & 1.2 & 1.7 & 1.1 \\
$2013-02-08$ & 1347.5 & 200 & Eff & 1046 & 0.5 & 1.7 & 0.1 \\
$2013-02-09$ & 1347.5 & 200 & Eff & 3821 & 2.0 & 2.0 & 1.4 \\
$2013-02-10$ & 1347.5 & 200 & Eff & 2339 & 1.7 & 1.8 & 1.3 \\
\hline Total & & & & 99444 & 24.8 & 28.3 & 16.0 \\
\hline
\end{tabular}

Notes. The value $v$ stands for the center frequency, BW for the bandwidth, $N_{\mathrm{GP}}$ for the number of extracted GPs, $T_{\text {radio }}$ and $T_{\mathrm{MAGIC}}$ indicate the duration of the radio and the corresponding VHE $\gamma$-rays observation respectively. The acronym Eff stands for Effelsberg radio telescope while WSRT for Westerbork Synthesis Radio Telescope.

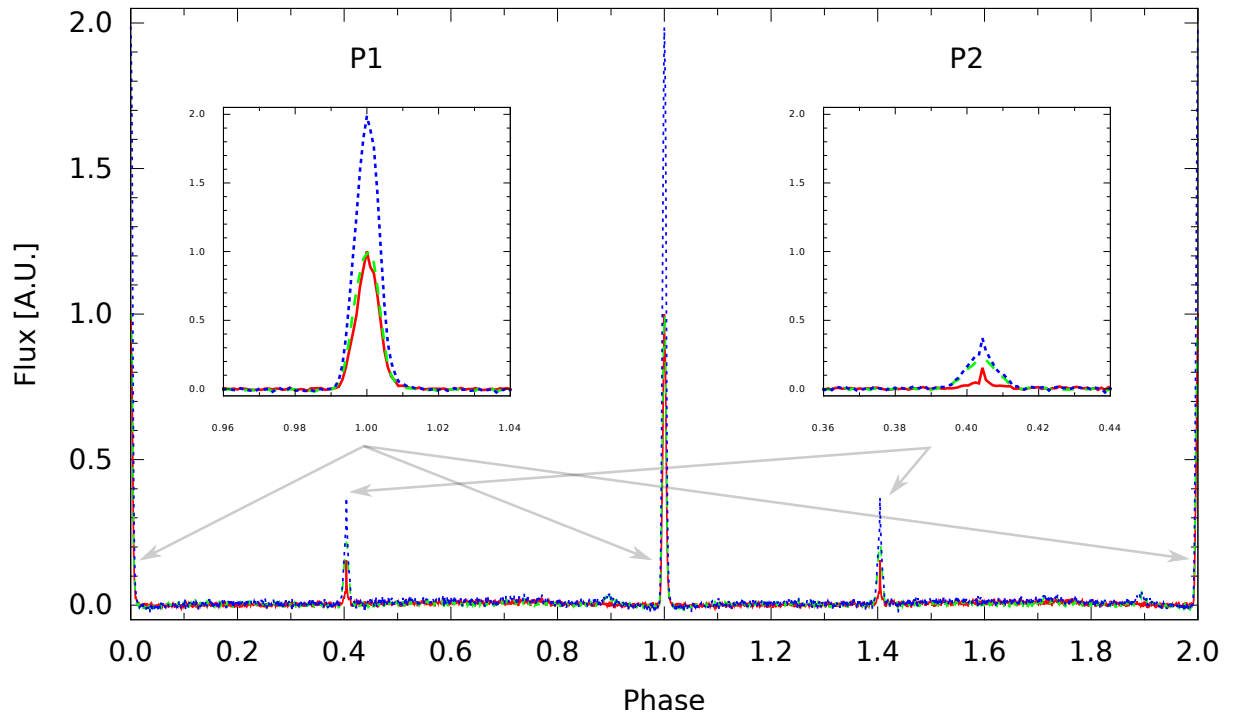

Fig. 1. Phase diagram resulting from one Effelsberg observation (2017-12-07, red solid curve) and one WSRT observation (2017-1210 , green dashed curve). MP is visible near phase 0.0 and 1.0 whereas LFIP near phases 0.4 and 1.4. The blue dotted curve represents the sum of both observations. were optimized to maximize the significance of the pulsed $\gamma$ ray signal, taking the continuous emission from the Crab Nebula as described in Aleksić et al. (2012) into account.

After optimizing the cuts in each energy bin separately we picked the bins in the energy range from 42.9 to $367.8 \mathrm{GeV}$ that correspond to the energy range in Aleksić et al. (2012). The $16 \mathrm{~h}$ of VHE $\gamma$-ray data taken simultaneously with radio observations led to a detection of the pulsar clearly above the background of the Crab Nebula (with $6.0 \sigma$ significance) in that range, as shown in Fig. 2. For the barycentering of the VHE $\gamma$-ray data the TEMPO2 pulsar timing software (Hobbs et al. 2006) and the same ephemeris files were used as for the radio data (Lyne et al. 1993). The folded light curve obtained after the barycentering process and the selection cuts are shown in Fig. 2: the gray shadowed areas are the results from a previous MAGIC phase resolved analysis of the Crab pulsar (Aleksić et al. 2012). The overlap with the present data (blue filled area) shows the compatibility between the two analyses, even if the energy ranges for the two results are slightly different (our results are shown here for the energy range from 43 to $368 \mathrm{GeV}$ while results from Aleksić et al. 2012 were obtained in the energy range from 50 to $400 \mathrm{GeV}$ ). To further quantify the compatibility with previous MAGIC results, we report in Table 3 the number of excess events, significance and the full width at half maximum (FWHM) of a Gaussian fit to the peaks of P1 and P2 respectively, from the work of Aleksić et al. (2012, 2014), Ansoldi et al. (2016) and compare those with our results.

\section{Correlation study}

\subsection{Approach}

Due to the lack of statistical methods for the correlation analysis of independent event lists ${ }^{2}$, but also for comparability with

2 A discussion of that problem can be found in Edelson \& Krolik (1988). 


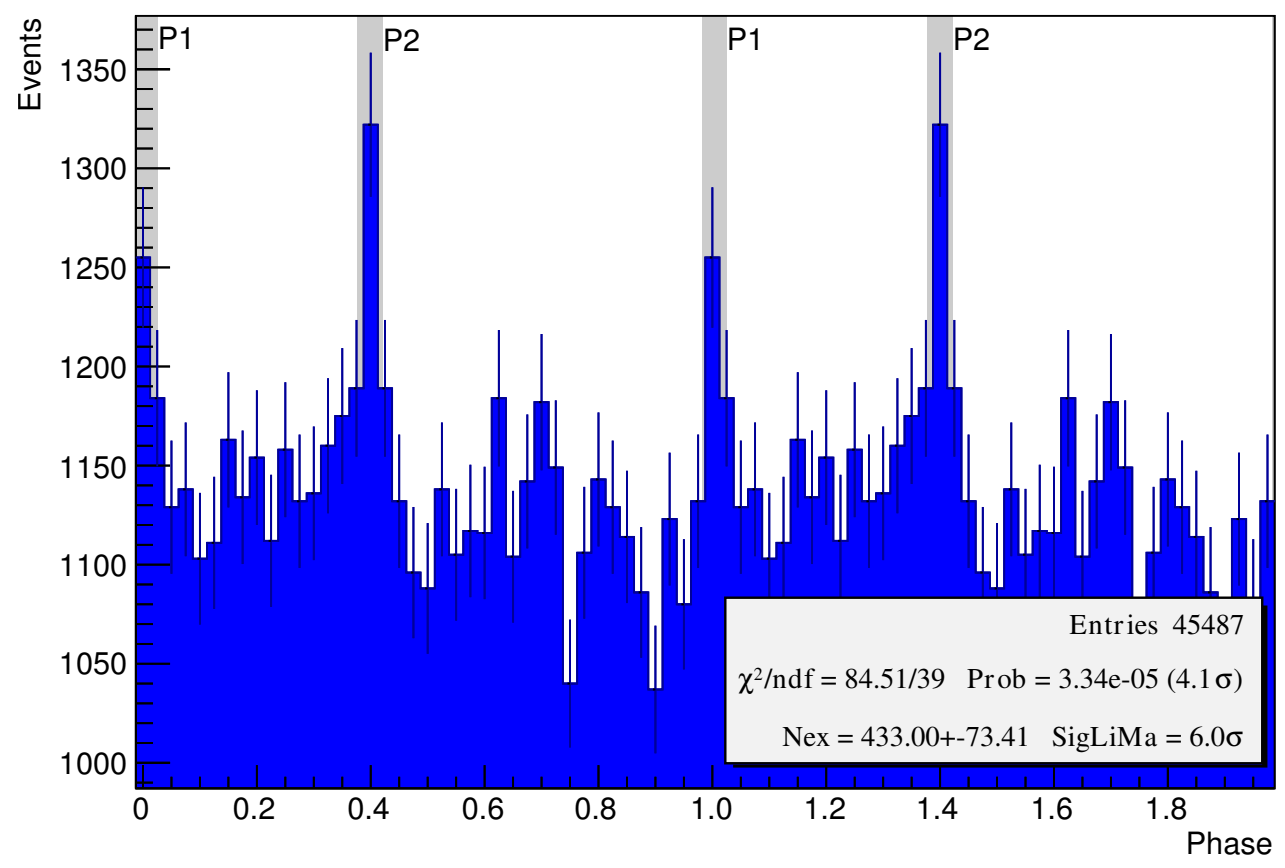

Fig. 2. Phase diagram resulting from the MAGIC data after the barycentering process and the cut selection, in the energy range $43-368 \mathrm{GeV}$. The resulting significance is $6 \sigma$. The gray areas correspond with the pulsed emission regions as determined by Aleksić et al. (2012) for the energy range $50-400 \mathrm{GeV}$. P1 is visible around phase values of 0 and 1 , whereas P2 is located around 0.4 and 1.4 .

results from previous studies with IACT data, we adopted the approach described in Aliu et al. (2012). The number of coincidences between VHE $\gamma$-rays and GPs was counted inside a given search window (SW, see Fig. 3). SWs were defined in terms of fractions or multiples of one rotational period of the Crab pulsar, namely: 1/9, 1/3, 1, 3, 9, 27, 81, 243, 729, 2187. With the aim of reducing the background emission from the Crab Nebula in our analysis, and to conduct dedicated studies on P1 and $\mathrm{P} 2$ respectively, we extended the approach of Aliu et al. (2012) by adopting SWs smaller than one rotational period (1/9 and 1/3). Exploring different SWs allowed us to change the trade-off between statistical and systematic uncertainties. Moreover, the SWs smaller than one that we consider in the present work only contain one of the pulsed emission components, either $\mathrm{P} 1$ or $\mathrm{P} 2$, depending at which phase range the radio GPs are located. Hence, SWs smaller than one rotation period of the Crab pulsar describe here the increase of VHE photons centered on radio GPs from only one of the regular emission components instead of both, allowing us to perform two separate analyses focused on P1 GPs and P2 GPs. As explained in Sect. 1, the indication of different emission mechanisms of GPs in P1 and P2 makes the separated analysis an important tool to deeply investigate the possible coincidences between GPs at various phase ranges at different energies.

Since the emission mechanism of radio GPs is unknown, a delay in the generation of radio GPs and VHE photons cannot be excluded. Therefore the search windows were constructed for three different orientations in time: before, centered on, and after a radio GP (see Fig. 3). This way possible time delays between the generation of radio GPs and VHE $\gamma$-rays were included in the search procedure. The described approach results in a total of 30 correlation searches.

\subsection{Monte Carlo simulations}

\subsubsection{Radio simulations}

Two statistical properties of the radio data were reproduced in the MC simulations: the average phase profile which we modeled by two Gaussians and the interarrival time between subsequent GPs. We modeled the interarrival times directly from the observed separations.

The interval between successive GPs was calculated and stored in a list. The list of interarrival times derived from observations was used instead of an analytic exponential distribution for two reasons: (1) There were non-trivial deviations from the exponential distribution due to the phase bound occurrence of radio GPs; (2) There were deviations at large time separations (more than 50 rotation periods) due to the fact that the observations at both telescopes were interrupted by weather, data writeout and other technical constraints.

Due to time gaps within the radio data sets (introduced during data recording to produce data chunks which were shorter in time and thus easier to reduce off-line), all interarrival times longer than $30 \mathrm{~s}$ were excluded from the simulation. All interarrivals shorter than this threshold were stored in a list. In the MC simulation a random interarrival time was fetched from the above-described list instead of drawing from an analytic exponential distribution. The parameters of the average profile were obtained by fitting Gaussian distributions to the P1 and P2 components in the radio data. To increase the signal-to-noise-ratio, the fit was performed on all the radio data collected during this campaign, with the exception of the Effelsberg data sets from 2013-01-09 and 2013-02-07 since both were taken at different center frequencies (see Table 2). A more detailed explanation can be found in Lewandowska (2015).

\subsection{2. $\gamma$-ray simulations}

In order to asses the significance level of the correlation, we produced correlation-free $\gamma$-ray data and searched for a correlation with the real radio data. The synthetic data had to reflect all the statistical properties of the real data. We produced such a data set in the following way ${ }^{3}$ :

1. The rate of events (before selection by Hadroness or $\theta^{2}$ parameters) was converted into a cumulative distribution function (CDF) with bin widths of one second.

Additional details can be found in Appendix A. 
Table 3. Comparison of the current data set with previous MAGIC observations of the pulsed emission from the Crab Nebula.

\begin{tabular}{lcccccc}
\hline \hline Reference & Emission component & $N_{\text {excess }}$ & $F W H M$ & $\sigma$ & $\begin{array}{c}\text { Duration } \\
{[\mathrm{h}]}\end{array}$ & $\begin{array}{c}E_{\text {range }} \\
{[\mathrm{GeV}]}\end{array}$ \\
\hline Aleksić et al. (2012) & $\mathrm{P} 1+\mathrm{P} 2$ & $1175 \pm 116$ & - & 10.4 & 73 & $46-416$ \\
\hline Aleksić et al. (2014) & $\mathrm{P} 1$ & $930 \pm 120$ & $0.025 \pm 0.007$ & 8 & 135 & $50-400$ \\
& $\mathrm{P} 2$ & $1510 \pm 120$ & $0.026 \pm 0.004$ & 12 & 135 & $50-400$ \\
\hline Ansoldi et al. (2016) & $\mathrm{P} 1$ & $1252 \pm 442$ & $0.010 \pm 0.003$ & 2.86 & 320 & $100-400$ \\
& $\mathrm{P} 2$ & $2537 \pm 454$ & $0.040 \pm 0.009$ & 5.66 & 320 & $100-400$ \\
\hline \multirow{2}{*}{ This work } & $\mathrm{P} 1+\mathrm{P} 2$ & $433 \pm 73$ & - & 6.06 & 16 & $43-368$ \\
& $\mathrm{P} 1$ & $144 \pm 41$ & $0.015 \pm 0.005$ & 3.5 & 16 & $43-368$ \\
& $\mathrm{P} 2$ & $289 \pm 58$ & $0.036 \pm 0.009$ & 4.9 & 16 & $43-368$ \\
\hline
\end{tabular}

2. A uniform random number was drawn and the first bin in the CDF was located where the fraction of events exceeds that random number.

3. A second uniform random number was drawn in order to determine a time stamp in the one second interval covered by the bin.

4. The time stamp obtained in step 3 does not yet reflect the fact that the VHE $\gamma$-ray data contains the pulsations from the Crab pulsar. Therefore, the time stamp was slightly modified in the following way: the event stayed within the same pulsar period, but the phase inside the rotation was drawn from a model containing a uniform background and two Gaussian peaks. This model was obtained by fitting the observed pulsed profile (after Hadroness and $\theta^{2}$ cuts). The adjusted phase was then converted back into a time value using the Taylor expansion formula (Eq. (8.4) in Lorimer \& Kramer 2012).

5 . The steps 2,3 , and 4 were repeated $M$ times. For each MC data set, $M$ was drawn randomly from a Poisson distribution with a mean of $N_{\text {proc }}$, where $N_{\text {proc }}$ is the total number of events after Hadroness and $\theta^{2}$ cuts. This way, one can get a synthetic uncorrelated VHE $\gamma$-ray data set with $M$ events.

To calculate confidence intervals with sufficiently low statistical error, 200 different synthetic VHE $\gamma$-ray data sets were produced by repeating the above procedure. As shown below in Sect. 3.3, we did not find a statistically significant correlation. Therefore we calculated and report upper limits to the degree of correlation. For this purpose we defined a correlation parameter $\kappa$, which is the fraction of $\gamma$-ray events arriving simultaneously to an observed GP. Using this parameter we also generated synthetic correlated $\gamma$-ray data sets with different values of the parameter $\kappa$. At first we generated a uncorrelated $\gamma$-ray signal using the described procedure, but the arrival times of $\kappa \cdot N_{\text {pulse }}$ events were replaced by randomly picked arrival times of radio GPs, $N_{\text {pulse }}$ being the number of detected pulsed events in the real VHE $\gamma$-ray data.

\subsection{Results}

The number of coincidence events in the observational data for different SWs are shown in Fig. 4, together with the uncorrelated simulation results (top) and the perfectly correlated $(\kappa=1)$ simulation results (bottom). Error bars for simulation results are obtained as a $1 \sigma$ fluctuation among 200 data sets (see Sect. 3.2).

As can be deduced from the top panel of Fig. 4, the observed enhancement of VHE $\gamma$-rays becomes higher for shorter search windows centered on a GP, though the bottom panel shows the correlation is well below $100 \%$. Therefore, only the number of VHE photons in a search window centered on a radio GP for a window length of 1/9, 1/3, 1 and $3 \mathrm{Crab}$ pulsar rotation periods

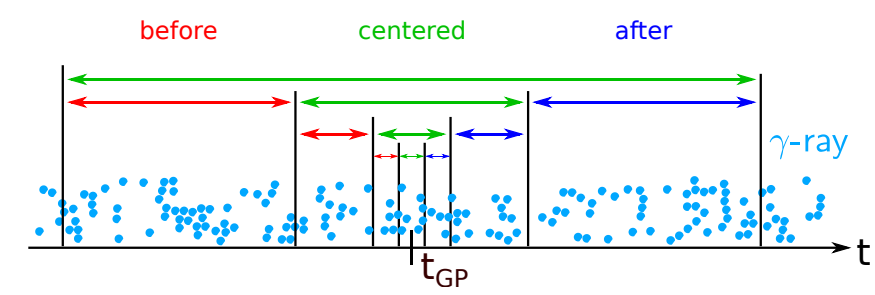

Fig. 3. Construction of search windows around a radio GP. The central window is symmetric around the arrival time of a radio GP. The advanced and delayed windows have the same length and are adjacent in time to the centered window. This construction arranges the search windows in a hierarchy where all three search windows of one length together form the centered search window of the next larger duration.

are examined in the forthcoming part of the analysis. To determine the enhancement quantitatively, MC simulations with different $\kappa$ values are compared with the corresponding data point. The results are shown in the right hand plots of Fig. 5.

The cyan line represents the data point from the respective search window. The red ticks correspond to the average values of the VHE $\gamma$-ray MC simulations for different values of $\kappa$. The $1 \sigma$ range around the average values is indicated by the vertical red bars as well as the green lines. The blue lines stand for the $1.96 \sigma$ range. Since a linear scaling of both the average and the upper and lower limits are expected, the plot also contains fitted linear trend lines as dashed curves. To determine the best estimated value of $\kappa$ which reflects the enhancement of VHE $\gamma$-rays seen in the data, we calculate the intersection of the horizontal cyan data line with the linear trend lines ${ }^{4}$. This procedure is carried out for all four search window lengths. The corresponding results are given in the upper four rows of Table 4 . The most significant deviation of $\kappa_{\text {best }}$ is seen at a search window of $1 / 9$ of the rotation period, which is in accordance with Fig. 4 . Since none of the $\kappa_{\text {best }}$ is significantly larger than $0,95 \%$ level upper limits on $\kappa$ are also calculated on each window size, as the intersection between the cyan and blue lines in the figure. They are shown in the column of " $\mathrm{CI}_{95 \%}$ " in Table 4.

In order to differentiate between P1 and P2 GPs (which correspond in this particular analysis to MP and LFIP as discussed in Sect. 1), we carry out the same analysis with radio GPs only within the phase ranges between -0.02 and 0.02 (centered on MP radio phase) and between 0.37 and 0.42 (centered on LFIP radio phase, see Table 1 ). The corresponding results are shown in Figs. 6 and 7 and are summarized in Table 4.

\footnotetext{
4 In one case $(\mathrm{SW}=3)$ this required extrapolation to $\kappa>0.5$, beyond the range for which MCMC simulations were performed.
} 
Completely Uncorrelated, $\mathrm{k}=0$
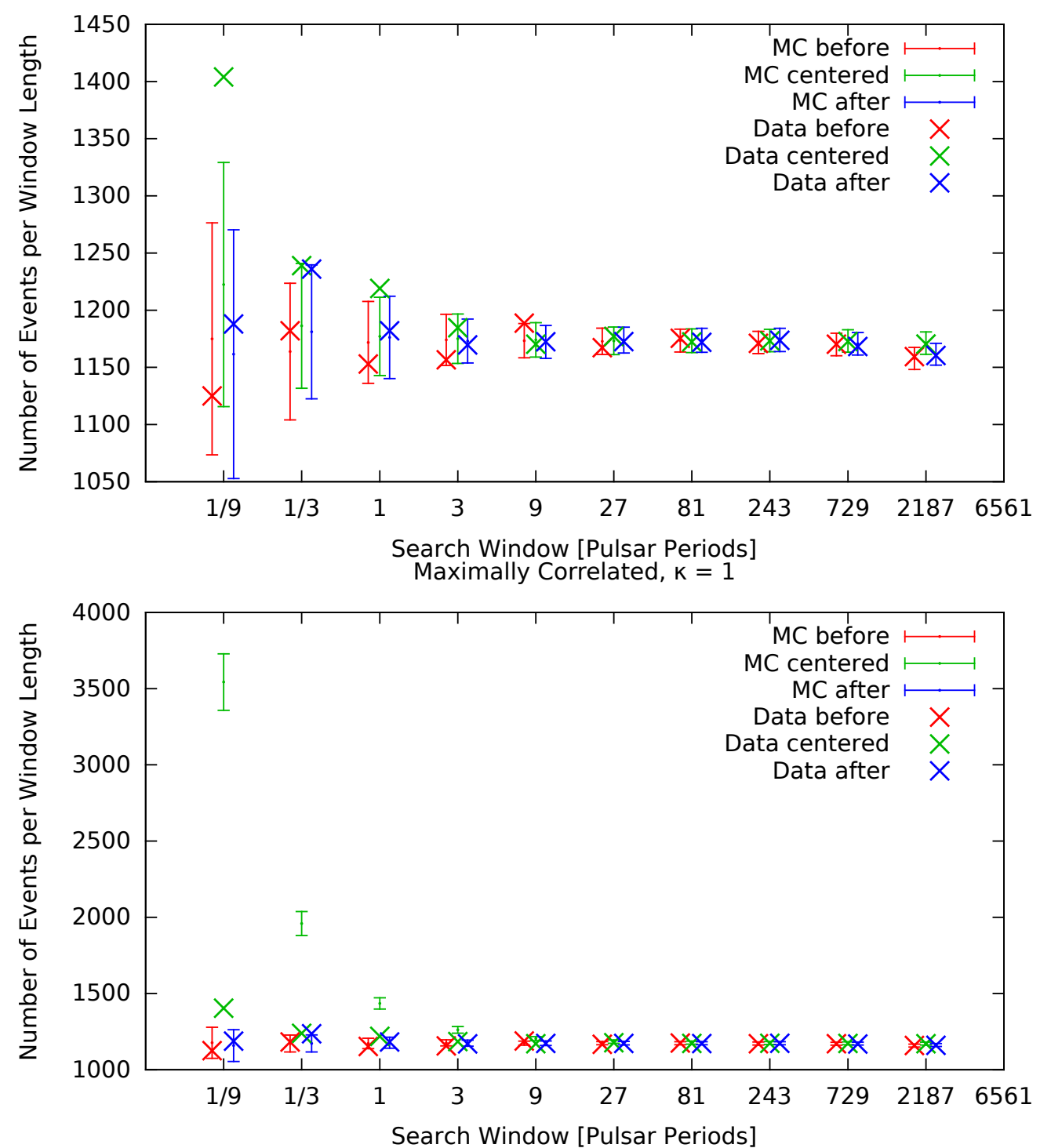

Fig. 4. Enhancement of VHE photons around occurring radio GPs resulting out of data sets (marked with crosses) and VHE $\gamma$-ray MC simulations (indicated by bars). Upper plot: results for a perfectly uncorrelated VHE $\gamma$-ray signal in the MC simulations $(\kappa=0)$, while lower plot: flux enhancement results for an injected VHE $\gamma$-ray signal which is perfectly correlated with GPs resulting from the radio data in a centered search window $(\kappa=1)$. The latter plot shows more clearly an increase of the number of VHE $\gamma$-rays centered on radio GPs for shorter search windows resulting from the data sets, indicating that the correlation is located at $\kappa<1$.

\section{Discussion}

The present results do not show a statistically significant correlation between radio GPs and VHE $\gamma$-rays from the Crab pulsar. No correlation was found also in several studies carried out in the past, including the work of Argyle et al. (1974), Lundgren et al. (1995), Bilous et al. (2011), Mickaliger et al. (2012), and Aliu et al. (2012). A correlation with optical photons was found by Shearer et al. (2003), as a 3\% higher average intensity over many periods with GPs observed. In order to compare this study with previous ones, it is useful to convert $\kappa$ to the factor of flux enhancement during GPs. It can approximately be done as follows. Upper limits in number of $\gamma$-rays accompanied with a radio GP $\left(N_{\mathrm{UL}}\right)$ are

$N_{\mathrm{UL}}=\kappa_{\mathrm{UL}} \cdot N_{\gamma}$,

where $N_{\gamma}$ is the number of observed pulsed $\gamma$-rays which is $443.0 \pm 73.4$ as shown in Fig. 2. Total observation time "around
GPs" $T_{\mathrm{GP}}$ can be computed from the number of obtained GPs $N_{\mathrm{GP}}$ and the size of the search window $T_{\mathrm{SW}}$ as

$T_{\mathrm{GP}}=N_{\mathrm{GP}} \cdot T_{\mathrm{SW}}=N_{\mathrm{GP}} \cdot P_{\mathrm{Crab}} \cdot f_{\mathrm{SW}}$,

where $f_{\mathrm{SW}}$ is the search window in fraction of the rotation period, such as $1 / 9,1 / 3,1$ and 3 for this study. Since $N_{\mathrm{GP}}$ is 99444 as shown in Table $2, T_{\mathrm{GP}} \simeq 0.93 \cdot f_{\mathrm{SW}} \mathrm{h}$.

$N_{\mathrm{UL}} / T_{\mathrm{GP}}$ should be compared with $N_{\gamma} / T_{\text {total }}$, where $T_{\text {total }}$ is the total observation time which is $16 \mathrm{~h}$. Then, the upper limit in the flux enhancement $F_{\mathrm{UL}}$ is written as

$$
\begin{aligned}
F_{\mathrm{UL}} & =\left(N_{\mathrm{UL}} / T_{\mathrm{GP}}\right) /\left(N_{\gamma} / T_{\text {total }}\right) \\
& =\frac{\left(\kappa \cdot N_{\gamma}\right) /\left(N_{\mathrm{GP}} \cdot P_{\mathrm{Crab}} \cdot f_{\mathrm{SW}}\right)}{N_{\gamma} / T_{\text {total }}} \\
& =17.3 \cdot \kappa / f_{\mathrm{SW}}
\end{aligned}
$$

Therefore, the upper limit in $\kappa$ of 0.45 for $f_{\mathrm{SW}}=1$ (see Table 4) translates to $740 \%$ of flux enhancement while $\kappa$ of 

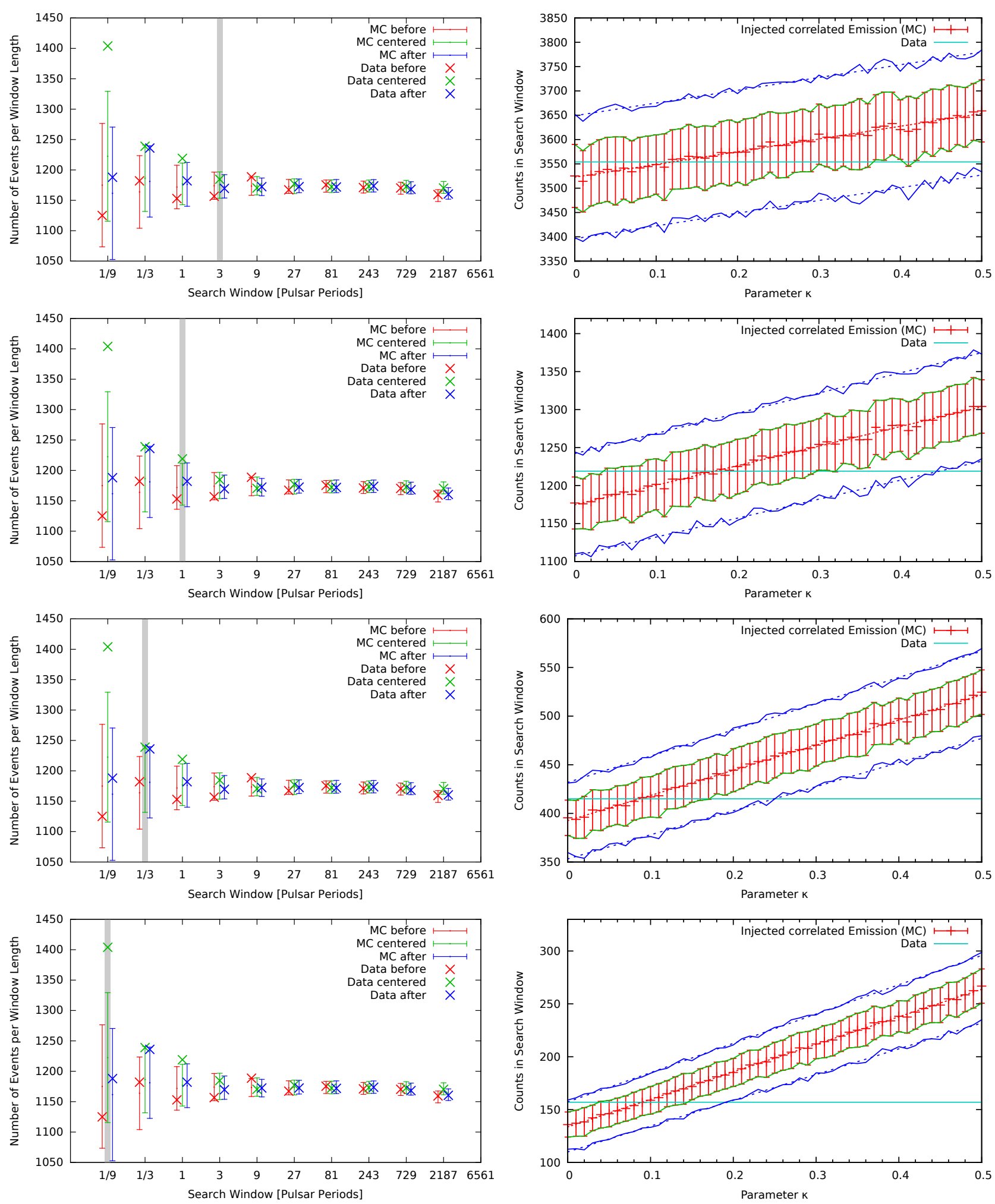

Fig. 5. From top to bottom: search windows of 3,1,1/3 and 1/9 Crab pulsar rotation periods length. Left: enhancements of VHE $\gamma$-rays before, centered on and after a radio GP. The gray bar indicates the search window for which the $\kappa$ dependence is studied in the respective right hand plot. Right: horizontal cyan line indicates the number of VHE $\gamma$-rays in the search window centered on GPs (the corresponding value from the observed data is indicated by a cross in the left hand plot. The normalization of the $y$-axis between the two columns is different). The plot also contains the results from 50 different sets of $\gamma$-ray MC simulations, using different values of $\kappa$. The average of each set is indicated by a red tick. The $1 \sigma$ range is indicated by the green lines, the $1.96 \sigma$ range (corresponding to a rejection of the null hypothesis on a $p=0.05$ confidence level) is indicated by the blue lines.

0.19 for $\mathrm{SW}=1 / 9$ translates to $2900 \%$. This calculation shows that the sensitivity at $\gamma$-ray energies and telescope time available for this study are not sufficient to detect a statistically significant correlation, or place an upper constraint comparable to the correlation observed in the optical regime. The corresponding expressions in Eq. (5) for the phase resolved analysis 
Table 4. Results of the correlation study between radio GPs and $\gamma$-rays that appear to be correlated with radio GPs (resulting from the intersection values between linear fits of $\gamma$-ray MC simulations and data points in the right hand figures of Figs. 6 and 7).

\begin{tabular}{lrrrrr}
\hline \hline Component & $\begin{array}{r}\mathrm{SW} \\
{\left[P_{\mathrm{Crab}}\right]}\end{array}$ & $\kappa_{\text {best }}$ & $\mathrm{CI}_{95 \%}$ & $p_{\text {err }}$ & $\begin{array}{r}F_{\mathrm{UL}} \\
{[\%]}\end{array}$ \\
\hline $\mathrm{ALL}$ & 3 & $0.12_{-0.12}^{+0.23}$ & 0.61 & 0.31 & 340 \\
$\mathrm{ALL}$ & 1 & $0.17_{-0.14}^{+0.14}$ & 0.45 & 0.11 & 740 \\
$\mathrm{ALL}$ & $1 / 3$ & $0.079_{-0.079}^{+0.079}$ & 0.24 & 0.14 & 1200 \\
$\mathrm{ALL}$ & $1 / 9$ & $0.086_{-0.048}^{+0.048}$ & 0.19 & 0.04 & 2900 \\
$(\mathrm{P} 1+\mathrm{P} 2)_{\gamma}$ & 3 & $0.074_{-0.074}^{+0.075}$ & 0.24 & 0.19 & 12 \\
$(\mathrm{P} 1+\mathrm{P} 2)_{\gamma}$ & 1 & $0.018_{-0.018}^{+0.043}$ & 0.11 & 0.38 & 17 \\
$(\mathrm{P} 1+\mathrm{P} 2)_{\gamma}$ & $1 / 3$ & $0.055_{-0.055}^{+0.032}$ & 0.13 & 0.06 & 60 \\
$\mathrm{P} 1_{r}$ & 3 & $0.08_{-0.08}^{+0.21}$ & 0.52 & 0.37 & 15 \\
$\mathrm{P} 1_{r}$ & 1 & $0.05_{-0.05}^{+0.12}$ & 0.30 & 0.35 & 25 \\
$\mathrm{P} 1_{r}$ & $1 / 3$ & $0.103_{-0.087}^{+0.087}$ & 0.30 & 0.10 & 76 \\
$\mathrm{P} 2_{r}$ & 3 & $0.129_{-0.055}^{+0.055}$ & 0.25 & 0.01 & 34 \\
$\mathrm{P} 2_{r}$ & 1 & $0.018_{-0.018}^{+0.029}$ & 0.09 & 0.27 & 36 \\
$\mathrm{P} 2_{r}$ & $1 / 3$ & $0.016_{-0.016}^{+0.022}$ & 0.07 & 0.25 & 85 \\
\hline
\end{tabular}

Notes. The first column indicates the used data sample without phase cuts (marked "ALL"), only P1 and P2 in the VHE $\gamma$-ray data + MCs (marked with a $\gamma$ ) and P1, P2 based on Gaussian fits in the radio data (marked with an " $r$ "). Their indices reflect whether the phase cuts are based on the radio, or $\gamma$-ray data. The acronym SW is standing for search window length, $P_{\text {Crab }}$ is the rotation period of the Crab pulsar, $\kappa_{\text {best }}$ is the intersection value, $\mathrm{CI}_{95 \%}$ is the upper value of the corresponding $95 \%$ confidence interval, $p_{\text {err }}$ the probability to obtain the observed number of events based on the mean and standard deviation of the MC simulations and $F_{\mathrm{UL}}$ the upper limit of the flux normalized to the pulsed VHE flux of the Crab pulsar.

are $F_{\mathrm{UL}, \mathrm{P} 1}=0.848 * \kappa / f$ and $F_{\mathrm{UL}, \mathrm{P} 2}=4.04 * \kappa / f$, accounting for the number of GPs (82 055 and 17041 , respectively) and the shortening of $T_{\text {total }}$ due to the phase cuts.

The only existing theoretical prediction for a correlation at frequencies higher than $5 \mathrm{GHz}$ is given by Lyutikov (2007). However, this model is applicable to radio GPs at the phase ranges of $\mathrm{P} 2$ above $5 \mathrm{GHz}$, which does not cover the frequency range of the observations presented in this work, making the model not applicable to our observations. The studies by Bilous et al. (2011) and Aliu et al. (2012) addressed radio GPs above $5 \mathrm{GHz}$, and reported $95 \%$ confidence level upper limits on the enhanced flux of five to ten times higher the flux measured by VERITAS. The higher energy threshold of VERITAS combined with the steep power-law spectrum of the Crab pulsar may have limited the sensitivity of the study. The correlation study carried out by Mickaliger et al. (2012) at $300 \mathrm{MHz}$ and $1.2 \mathrm{GHz}$ did not result in any statistically significant findings in spite of extended searches for coincidences between radio GPs and $\mathrm{GeV} \gamma$-rays. In the latter case data taken by the Large Area Telescope (LAT) on board the Fermi satellite (Abdo et al. 2009) were used which, in comparison with the present work, provided data with a lower background. The data set was spanning over 15 months but the smaller collection area of the space-borne detector could be a limiting factor regarding the number of detected events which might be the reason for not detecting any correlation. The Hitomi X-ray satellite also searched for a correlation between radio
GPs and soft X-rays. Aharonian (2018) report upper limits of $22 \%-80 \%$ of the peak flux at a range of $2-300 \mathrm{keV}$.

A recent review on the radio emission physics of the Crab pulsar given by Eilek \& Hankins (2016) suggests that the observed radio and high energy emission might have origin in the same spatial regions within the magnetosphere, due to the fact that both, main radio and high energy emission components, appear approximately at the same phase ranges. However, a satisfactory theoretical approach still needs to be found. The variety of instabilities in the radio emission of the Crab pulsar (including GPs) leads to the assumption that the radio emission sites are dynamic and unstable (Eilek \& Hankins 2016). The connection between these regions and the high energy emission is still an open question. Since the Crab pulsar has been an object of regular monitoring campaigns at radio (Lyne et al. 1993) as well as at VHE $\gamma$-ray wavelengths (Meyer et al. 2010), we suggest a coordination of the respective observations. Simultaneous observations at both energy ranges can lead to a further examination of the obtained results, especially below and above $5 \mathrm{GHz}$ by including radio GPs from the Crab pulsar at frequencies before and after the described transition.

\section{Summary}

In this work a correlation study between radio GPs and $\gamma$-rays above $60 \mathrm{GeV}$ from the Crab pulsar is presented. The data used for this study were taken with the Effelsberg radio telescope (at $1347.5 \mathrm{MHz}$ and $1410 \mathrm{MHz}$ ), the WSRT (at $1380.0 \mathrm{MHz}$ ) and the MAGIC telescopes (Figs. 1 and 2). The total overlap between the radio and $\gamma$-ray observations (excluding all gaps which are longer than $30 \mathrm{~s}$ ) results in $16 \mathrm{~h}$ (see Table 2). The approach for our correlation search is based on the idea described in Aliu et al. (2012), consisting of the construction of search windows around the arrival time of each radio GP resulting from the radio data (see Fig. 3). We compare the amount of VHE $\gamma$-rays around a radio GP resulting from the observational data and MC simulations which are based on the timing characteristics of the data. To estimate the degree of correlation, we inject a variable level of a signal which is perfectly correlated with radio GPs into the simulations. With this approach we determine the fraction of VHE photons which appear to be correlated with radio GPs (indicated by component "ALL" and denoted as $\kappa_{\text {best }}$ in Table 4 ).

Based on the described study, we conclude the following:

- No statistically significant correlation between VHE pulsed photons and radio GPs at $1.4 \mathrm{GHz}$ was found for search window sizes of $1 / 9,1 / 3,1$, and 3 times the rotation period.

- The most stringent upper limit in the correlation degree was obtained for the search window of $1 / 9$ of the rotational period, and not more than $19 \%$ of the $\gamma$-rays are accompanied by GPs. This corresponds to an upper limit on the increase in pulsed flux of no more than $2900 \%$ at $95 \%$ confidence level.

- GPs in MP and LFIP are separately analyzed, and the corresponding upper limits are presented in Table 4. Converting the correlation to a flux enhancement relative to the pulsed flux, we find upper limits between $15 \%$ (P1 phase cut, search window of $3 \mathrm{P}_{\mathrm{Crab}}$ ) and $85 \%$ ( $\mathrm{P} 2$ phase cut, search window of $\left.1 / 3 \mathrm{P}_{\text {Crab }}\right)$. The phase cuts do allow to place more stringent upper limits, but no statistically significant correlation could be found.

Future observations with a larger overlap or higher sensitivity, as hopefully provided by the Cherenkov Telescope Array (CTA, Acharya et al. 2013; CTA Consortium 2019), will help to provide further constraints in the still open question of a correlation 

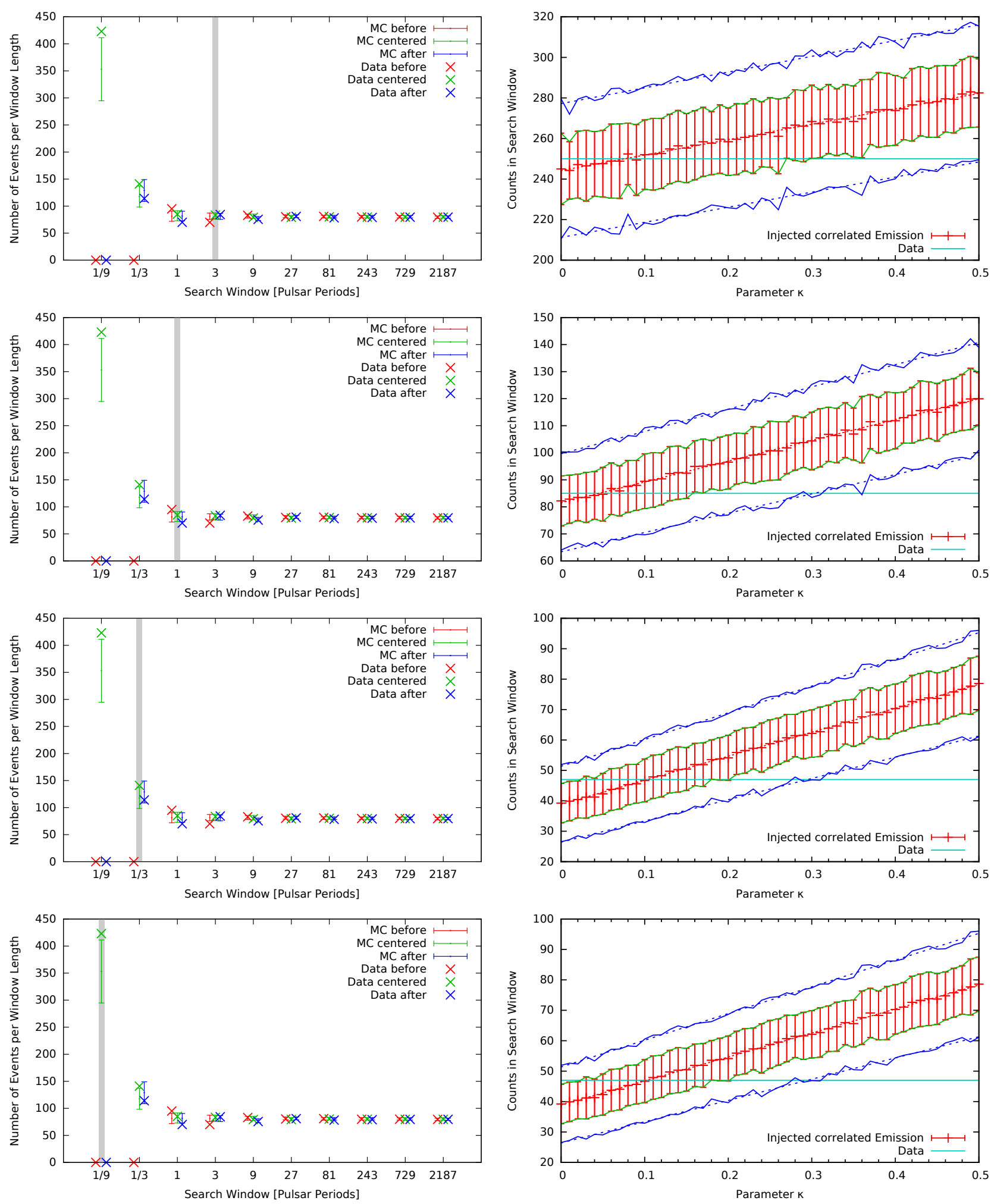

Fig. 6. Results as described in Fig. 5 for the P1 emission component (MP radio phase). The MC error bars in the left hand part of this figure were computed for $\kappa=0$ and not for the best fit $\kappa$ value.

between radio GPs and the VHE $\gamma$-ray emission from the Crab pulsar.

Acknowledgements. NL would like to thank Axel Jessner (MPIfR), Jean Eilek (NRAO), Maura McLaughlin (WVU), Ryan Lynch (GBO) and Vlad Kondratiev (ASTRON) for numerous helpful comments which improved the quality of the paper. We acknowledge Marina Manganaro (Croatian MAGIC Consortium) for her continuous help in preparing the manuscript. We would like to thank the anonymous referee for numerous comments which helped us to improve the quality of the manuscript. NL would also like to thank Ramesh Karuppusamy (MPIfR), Alex Kraus (MPIfR), Ralf Kisky (MPIfR), Jörg Barthel (MPIfR) and Thomas Wedel (MPIfR) for constant support during the observations with the Effelsberg radio telescope. NL gratefully acknowledges the 
M. L. Ahnen et al.: Statistics of VHE $\gamma$-rays in temporal association with radio giant pulses from the Crab pulsar
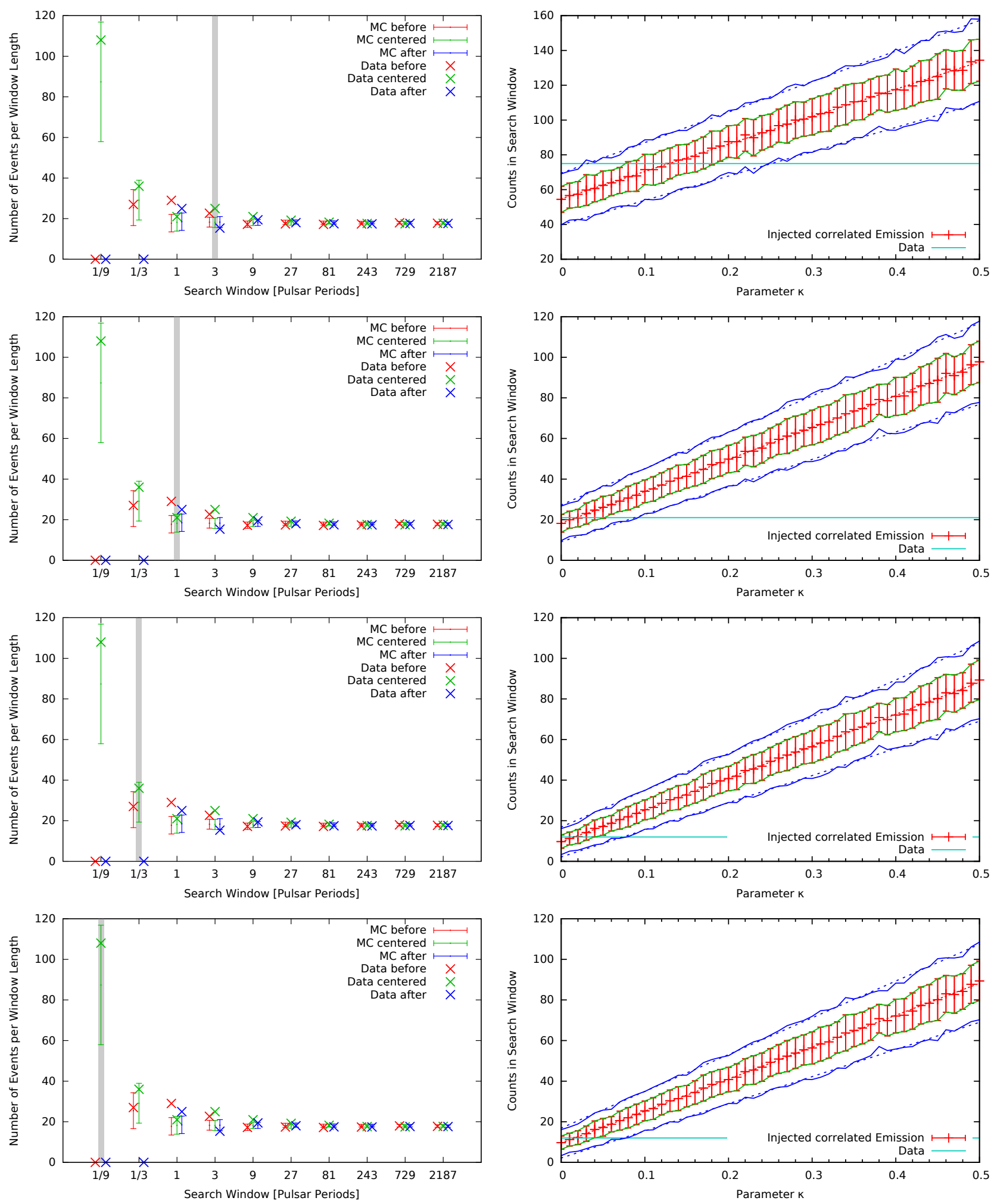

Fig. 7. Results as described in Figs. 5 and 6 for the P2 emission component (LFIP radio phase).

support of this study by the ASTRON/JIVE Helena Kluyver female visitor program. This study is partly based on observations with the $100-\mathrm{m}$ telescope of the MPIfR (Max-Planck-Institut für Radioastronomie) at Effelsberg. Another part of this study was carried out with data taken with the Westerbork Synthesis Radio Telescope (WSRT). The Westerbork Synthesis Radio Telescope is operated by the ASTRON (Netherlands Institute for Radio Astronomy) with support from the Netherlands Foundation for Scientific Research (NWO). We would like to thank the Instituto de Astrofísica de Canarias for the excellent working conditions at the Observatorio del Roque de los Muchachos in
La Palma. The financial support of the German BMBF and MPG, the Italian INFN and INAF, the Swiss National Fund SNF, the ERDF under the Spanish MINECO (FPA2015-69818-P, FPA2012-36668, FPA2015-68378-P, FPA201569210-C6-2-R, FPA2015-69210-C6-4-R, FPA2015-69210-C6-6-R, AYA201571042-P, AYA2016-76012-C3-1-P, ESP2015-71662-C2-2-P, CSD2009-00064), and the Japanese JSPS and MEXT is gratefully acknowledged. This work was also supported by the Spanish Centro de Excelencia "Severo Ochoa" SEV-20120234 and SEV-2015-0548, and Unidad de Excelencia "María de Maeztu" MDM2014-0369, by the Croatian Science Foundation (HrZZ) Project 09/176 and the 
University of Rijeka Project 13.12.1.3.02, by the DFG Collaborative Research Centers SFB823/C4 and SFB876/C3, the Polish National Research Centre grant UMO-2016/22/M/ST9/00382 and by the Brazilian MCTIC, CNPq and FAPERJ.

\section{References}

Abdo, A. A., Ackermann, M., Ajello, M., et al. 2009, ApJS, 183, 46 Abdo, A. A., Ackermann, M., Ajello, M., et al. 2010, ApJ, 708, 1254 Acharya, B. S., Actis, M., Aghajani, T., et al. 2013, Astropart. Phys., 43, 3 Aleksić, J., Alvarez, E. A., Antonelli, L. A., et al. 2012, A\&A, 540, A69 Aleksić, J., Ansoldi, S., Antonelli, L. A., et al. 2014, A\&A, 565, L12 Aleksić, J., Ansoldi, S., Antonelli, L. A., et al. 2016, Astropart. Phys., 72, 76 Aliu, E., Anderhub, H., Antonelli, L. A., et al. 2008, Science, 322, 1221 Aliu, E., Archambault, S., Arlen, T., et al. 2012, ApJ, 760, 136

Ansoldi, S., Antonelli, L. A., Antoranz, P., et al. 2016, A\&A, 585, A133

Argyle, E., \& Gower, J. F. R. 1972, ApJ, 175, L89

Argyle, E., Baird, G., Grindlay, J., Helmken, H., \& Omongain, E. 1974, Nuovo Cimento B Serie, 24, 153

Baring, M. G. 2004, AdSpR, 33, 552

Bilous, A. V., Kondratiev, V. I., McLaughlin, M. A., et al. 2011, ApJ, 728, 110

Bilous, A. V., McLaughlin, M. A., Kondratiev, V. I., \& Ransom, S. M. 2012, ApJ, 749, 24

Casse, J. L., Woestenburg, E. E. M., \& Visser, J. J. 1982, IEEE Trans. Microwave Theory Tech., 30, 201

Cerutti, B., Werner, G. R., Uzdensky, D. A., \& Begelman, M. C. 2012, in American Institute of Physics Conference Series, eds. F. A. Aharonian,

W. Hofmann, \& F. M. Rieger, 1505, 631

Contopoulos, I. 2007, A\&A, 472, 219

Contopoulos, I., \& Kalapotharakos, C. 2010, in 9th International Conference of the Hellenic Astronomical Society, eds. K. Tsinganos, D. Hatzidimitriou, \& T. Matsakos, ASP Conf. Ser., 424, 128

Contopoulos, I., Kazanas, D., \& Fendt, C. 1999, ApJ, 511, 351

CTA Consortium 2019, Science with the Cherenkov Telescope Array (World Scientific Publishing Co.)

Daugherty, J. K., \& Harding, A. K. 1982, ApJ, 252, 337

Edelson, R. A., \& Krolik, J. H. 1988, ApJ, 333, 646

Eilek, J. A., \& Hankins, T. H. 2016, J. Plasma Phys., 82, 635820302

Ellingson, S. W., Clarke, T. E., Craig, J., et al. 2013, ApJ, 768, 136

Fierro, J. M., Michelson, P. F., Nolan, P. L., \& Thompson, D. J. 1998, ApJ, 494, 734

Fomin, V. P., Stepanian, A. A., Lamb, R. C., et al. 1994, Astropart. Phys., 2, 137

Hankins, T. H. 2012, in Electromagnetic Radiation from Pulsars and Magnetars, eds. W. Lewandowski, O. Maron, \& J. Kijak, ASP Conf. Ser., 466

Hankins, T. H., \& Eilek, J. A. 2007, ApJ, 670, 693

Hankins, T. H., \& Rickett, B. J. 1975, Comput. Phys., 14, 55

Hankins, T. H., Kern, J. S., Weatherall, J. C., \& Eilek, J. A. 2003, Nature, 422, 141

Hankins, T. H., Rankin, J. M., \& Eilek, J. A. 2009, Astro 2010: The Astronomy and Astrophysics Decadal Survey, Astro2010 Science Frontier Panel Stars and Stellar Evolution, Science White Papers, no., 112

Hankins, T. H., Jones, G., \& Eilek, J. A. 2015, ApJ, 802, 130

Hankins, T. H., Eilek, J. A., \& Jones, G. 2016, ApJ, 833, 47

Harding, A. K. 2013, Front. Phys., 8, 679

Harding, A. K., Stern, J. V., Dyks, J., \& Frackowiak, M. 2008, ApJ, 680, 1378

Hewish, A., Bell, S. J., Pilkington, J. D. H., Scott, P. F., \& Collins, R. A. 1968 Nature, 217, 709

Hirotani, K. 2008, ApJ, 688, L25

Hitomi Collaboration (Aharonian, F., et al.) 2018, PASJ, 70, 15

Hobbs, G. B., Edwards, R. T., \& Manchester, R. N. 2006, MNRAS, 369, 655

Jessner, A., Popov, M. V., Kondratiev, V. I., et al. 2010, A\&A, 524, A60

Karuppusamy, R., Stappers, B., \& van Straten, W. 2008, PASP, 120, 191

Karuppusamy, R., Stappers, B. W., \& van Straten, W. 2010, A\&A, 515, A36

Kuiper, L., Hermsen, W., Cusumano, G., et al. 2001, A\&A, 378, 918

Lazarus, P., Karuppusamy, R., Graikou, E., et al. 2016, MNRAS, 458, 868

Lewandowska, N. 2015, PhD Thesis, Universität Würzburg

Lorimer, D. R., \& Kramer, M. 2012, Handbook of Pulsar Astronomy (Cambridge: Cambridge University Press)

Lundgren, S. C., Cordes, J. M., Ulmer, M., et al. 1995, ApJ, 453, 433

Lyne, A. G., Pritchard, R. S., \& Graham-Smith, F. 1993, MNRAS, 265, 1003

Lyutikov, M. 2007, MNRAS, 381, 1190

Manchester, R. N., Hobbs, G. B., Teoh, A., \& Hobbs, M. 2005, AJ, 129, 1993

Meyer, M., Horns, D., \& Zechlin, H.-S. 2010, A\&A, 523, A2

Mickaliger, M. B., McLaughlin, M. A., Lorimer, D. R., et al. 2012, ApJ, 760, 64

Mikami, R., Terasawa, T., Kisaka, S., et al. 2014, in Suzaku-MAXI 2014: Expanding the Frontiers of the X-ray Universe, eds. M. Ishida, R. Petre, \& K. Mitsuda, 180
Mikami, R., Terasawa, T., Takefuji, K., et al. 2013, in The Fast and the Furious: Energetic Phenomena in Isolated Neutron Stars, Pulsar Wind Nebulae and Supernova Remnants, ed. J. U. Ness, 58

Moffett, D. A., \& Hankins, T. H. 1996, ApJ, 468, 779

Oosterbroek, T., Cognard, I., Golden, A., et al. 2008, A\&A, 488, 271

Ruderman, M. A., \& Sutherland, P. G. 1975, ApJ, 196, 51

Shearer, A., Stappers, B., O'Connor, P., et al. 2003, Science, 301, 493

Spitkovsky, A. 2006, ApJ, 648, L51

Strader, M. J., Johnson, M. D., Mazin, B. A., et al. 2013, ApJ, 779, L12

Tan, G. H. 1991, in IAU Colloq. 131: Radio Interferometry. Theory, Techniques, and Applications, eds. T. J. Cornwell, \& R. A. Perley, ASP Conf. Ser., 19, 42 Tang, A. P. S., Takata, J., Jia, J. J., \& Cheng, K. S. 2008, ApJ, 676, 562 Tchekhovskoy, A., Spitkovsky, A., \& Li, J. G. 2013, MNRAS, 435, L1 Uzdensky, D. A., \& Spitkovsky, A. 2014, ApJ, 780, 3 van Straten, W., \& Bailes, M. 2011, PASA, 28, 1

VERITAS Collaboration (Aliu, E., et al.) 2011, Science, 334, 69

Zanin, R., Carmona, E., Sitarek, J., et al. 2013, Proceedings of the 33rd International Cosmic Ray Conference (ICRC2013): Rio de Janeiro, Brazil, July 2-9, 0773

1 ETH Zurich, 8093 Zurich, Switzerland

2 Università di Udine, and INFN Trieste, 33100 Udine, Italy

3 Japanese MAGIC Consortium: ICRR, The University of Tokyo, 277-8582, Chiba, Japan; Department of Physics, Kyoto University, 606-8502, Kyoto, Japan; Tokai University, 259-1292 Kanagawa, Japan

${ }^{4}$ National Institute for Astrophysics (INAF), 00136 Rome, Italy

5 Università di Padova and INFN, 35131 Padova, Italy

${ }^{6}$ Croatian MAGIC Consortium: University of Rijeka, Department of Physics, 51000 Rijeka, University of Split - FESB, 21000 Split, University of Zagreb - FER, 10000 Zagreb, University of Osijek, 31000 Osijek and Rudjer Boskovic Institute, 10000 Zagreb, Croatia

7 Saha Institute of Nuclear Physics, HBNI, 1/AF Bidhannagar, Salt Lake, Sector-1, Kolkata 700064, India

8 Max-Planck-Institut für Physik, 80805 München, Germany

${ }^{9}$ Centro Brasileiro de Pesquisas Físicas (CBPF), Urca, 22290-180 Rio de Janeiro (RJ), Brazil

${ }_{10}$ Universidad Complutense, 28040 Madrid, Spain

11 Inst. de Astrofísica de Canarias, 38200 La Laguna, and Universidad de La Laguna, Dpto. Astrofísica, 38206 La Laguna, Tenerife, Spain

12 University of Łódź, Department of Astrophysics, 90236 Łódź, Poland

13 Deutsches Elektronen-Synchrotron (DESY), 15738 Zeuthen, Germany

14 Humboldt University of Berlin, Institut für Physik Newtonstr. 15, 12489 Berlin, Germany

15 Also at Dipartimento di Fisica, Università di Trieste, 34127 Trieste, Italy

16 Institut de Física d'Altes Energies (IFAE), The Barcelona Institute of Science and Technology (BIST), 08193 Bellaterra, Barcelona, Spain

17 Università di Siena, and INFN Pisa, 53100 Siena, Italy

18 Technische Universität Dortmund, 44221 Dortmund, Germany

19 Universität Würzburg, 97074 Würzburg, Germany

${ }^{20}$ Finnish MAGIC Consortium: Tuorla Observatory and Finnish Centre of Astronomy with ESO (FINCA), University of Turku, Vaisalantie 20, 21500 Piikkiö, Astronomy Division, University of Oulu, 90014 Oulu, Finland

21 Departament de Física, and CERES-IEEC, Universitat Autónoma de Barcelona, 08193 Bellaterra, Spain

22 Universitat de Barcelona, ICC, IEEC-UB, 08028 Barcelona, Spain

23 Department of Physics and Astronomy, West Virginia University, Morgantown, WV 26506, USA and Center for Gravitational Waves and Cosmology, West Virginia University, Chestnut Ridge Research Building, Morgantown, WV 26505, USA

24 Inst. for Nucl. Research and Nucl. Energy, Bulgarian Academy of Sciences, 1784 Sofia, Bulgaria

25 INAF-Trieste and Dept. of Physics \& Astronomy, University of Bologna, Bologna, Italy

26 Università di Pisa, and INFN Pisa, 56126 Pisa, Italy

27 ASTRON, PO Box 2, 7990 Dwingeloo, The Netherlands 


\section{Appendix A: Description of Monte Carlo simulations}

The interpretation of the flux enhancements critically relies on the prediction of coincident radio and $\gamma$-ray counts from uncorrelated events. The extraction of statistical features of the present radio and $\gamma$-ray observations, as well as the construction of the simulated MC observations (which have the same statistical properties but are uncorrelated by construction), are here described step by step. Afterward, the $\gamma$-ray events which are coincident with radio GPs in some short search window can be regarded as real, potentially correlated, observations.

\section{A.1. Determination of statistical properties - radio observations}

\section{A.1.1. Event count}

The first property, which is matched by synthetic observation, is the total number of observed radio GPs. This number is determined for each night of observation and is designated by $N$ (it is important to note that there is no relation with the quantity $N$ from Sect. 3.2.2).

\section{A.1.2. Phase profile}

The second reproduced property is the phase bound occurrence of GPs. GPs have been observed only at the phase ranges of P1 and P2. The distribution of GPs is modeled by a Gaussian function. Since the observational GP data used for this study only includes single pulses brighter than five times the rms (seven times the rms in the case of Effelsberg data) of the raw data, they contain few pulses outside of the average emission components. One set of statistical parameters for each observing night are the amplitude $a$, the phase $m$ and the width $s$ for both regular emission components $\mathrm{P} 1$ and $\mathrm{P} 2$. The data are then modeled by the probability $p$ that a GP arrives at a rotational phase $\varphi$.

$p(\varphi)=\frac{a_{1}}{\sqrt{2 \pi} s_{1}} \exp \left(-\frac{1}{2} \frac{\left(\varphi-m_{1}\right)^{2}}{s_{1}^{2}}\right)+\frac{a_{2}}{\sqrt{2 \pi} s_{2}} \exp \left(-\frac{1}{2} \frac{\left(\varphi-m_{2}\right)^{2}}{s_{2}^{2}}\right)$

The normalization of $\mathrm{p}$ is such that the integral over all probabilities $\int_{0}^{1} p(\varphi) \mathrm{d} \varphi=N$, where $N$ is the total number of GPs observed in the respective night. The rotational phase $\varphi$ is restricted to the range $0 \ldots 1$ and the probability density is aliased to this range. Hence any remaining nonzero probability for the arrival at $\varphi=1.01$ is added to the probability for an arrival at rotational phase $\varphi=0.01$ (of the next rotation). The fitting procedure which is used to determine values of $a, m$ and $s$ from the observed data, operates on binned rotational phases $\varphi$ with 1000 bins for the interval $0 \ldots 1$. The procedure is first done jointly for all observations that were carried out with one telescope at one frequency (see Table 1 for details). The results of this first fit, made robust by the large number of available events, are then used as starting parameters for the individual fitting of all observation nights.

\section{A.1.3. Interarrival times}

The third set of statistical properties represented is the distribution of interarrival times between successive GPs. The interarrival times are modeled directly from the observed separations.
To be able to do this, the interval between successive GPs was calculated and stored in a list. Excessively large intervals, above $30 \mathrm{~s}$, for example, were discarded. The usage of the list of interarrival times in the Markov Chain Monte Carlo (MCMC) simulation of synthetic observations is described below.

\section{A.2. Determination of statistical properties $-\gamma$-ray observations}

\section{A.2.1. Phase profile}

The $\gamma$-ray observations employed in the present work have a substantially higher level of background emission and therefore need to be modeled differently. The distribution of $\gamma$-ray events over rotational phase is very similar to the respective distribution for radio GPs, but includes a constant background a3:

$p(\varphi)=\frac{a_{1}}{\sqrt{2 \pi} s_{1}} \exp \left(-\frac{1}{2} \frac{\left(\varphi-m_{1}\right)^{2}}{s_{1}^{2}}\right)+\frac{a_{2}}{\sqrt{2 \pi} s_{2}} \exp \left(-\frac{1}{2} \frac{\left(\varphi-m_{2}\right)^{2}}{s_{2}^{2}}\right)+a 3$

To determine all parameters in this equation, all observed $\gamma$-ray events are binned into 200 phase bins and fitted with the above equation.

\section{A.2.2. Event counts}

We refer to the fact that the total number of events $N$ is split into a number of "background" events

$N_{\text {off }}=\int_{0}^{1} a 3 \mathrm{~d} \varphi$

and "pulsed" events

$N_{1,2}=\int \frac{a_{1,2}}{\sqrt{2 \pi} s_{1,2}} \exp \left(-\frac{1}{2} \frac{\left(\varphi-m_{1,2}\right)^{2}}{s_{1,2}^{2}}\right) \mathrm{d} \varphi$

\section{A.2.3. Trigger rates}

The distribution of interarrival times in this case is dominated by background events and is therefore not as relevant as for the radio data. However, there is another effect which needs to be modeled in order to avoid systematic differences between real and synthetic observations generated from the MCMC simulation: the telescope performance varies according to zenith angles, moonlight and weather conditions. To take that into account, the raw trigger rate is determined for every one second interval and is later used to create events which follow the correct arrival rate over the course of the night. The raw trigger rate is used because of its stability with respect to discrete Poisson noise. While examining the data, we found that the fraction of $\gamma$-ray events is constant even during changes in the trigger rate.

\section{A.2.4. Identification of time windows with multi-wavelength coverage}

The observations at radio wavelengths and $\gamma$-rays show gaps in coverage due to unfavorable weather conditions, switches between sub-runs during data-taking and hardware problems. Consequently, only time windows during which observations at both wavelength ranges were successfully carried out can be 
used for our data analysis. The existence of gaps in the middle of the observing night might alter the overall statistics and therefore has to be modeled in the MCMC simulations as well. We assume that a gap exists in the multi-wavelength coverage when no radio GP or $\gamma$-ray event has been received for $30 \mathrm{~s}$. The currently open observing window is closed at the arrival time of the last event before the gap and a new window is tentatively started. If the length of a resulting window is below the cutoff length, it is omitted.

\section{A.3. Determination of the arrival rate of (nearly) coincident events}

The fundamental idea of the analysis method is to take a radio GP, take a small time window around it and to count the number of $\gamma$-ray events in that window. This is repeated for all radio GPs and the total number of $\gamma$-ray events in all such search windows is added up. At the end the total number is divided by the number of radio GPs and the duration of the search window to give an estimate of the $\gamma$-ray count rate, which is nearly coincident with radio GPs for the given width of the search window. Emission processes that lead to the production of both, radio GPs and $\gamma$-rays, but with some small time lag between the two, cannot be excluded. Therefore the analysis process does not only use one SW, centered around the arrival time of the radio GP, but two additional SWs of the same length. The "before" window covers the time span before the start of the centered window and the "after" window starts at the end of the centered window. See Fig. 3 for an illustration of the relative timing of the search windows. Other staggerings are imaginable, such as an "early" window which covers a time interval exactly up to the BAT (Barycentered Arrival Time) of the radio GP, or a "late" window that starts at this BAT. However, such staggering does not allow for the following implementation method that is used to efficiently compute the arrival rates for search windows of different length. The shortest SWs cover 1/9 of one pulsar rotation and there are separate "before", "centered" and "after" windows. The three SWs together are $1 / 3$ of a pulsar rotation and are centered around the arrival time of the radio GP. In other words, they cover exactly the centered SW for the duration of $1 / 3$ rotation. Together with the "before" and "after" windows of $1 / 3$ rotation each, one pulsar rotation is covered. This nested construction continues all the way along the centered window of the longest duration, 37 rotations. In total the ten different SWs durations require 21 nested windows, which can be computed during a single pass through the data. To output the arrival rates for a given duration, the windows of longer durations are ignored and all windows of shorter durations are summed to determine the number of events in the centered window. This method has reduced the analysis run time by nearly one order of magnitude, compared to an initial, more naive implementation that computed the arrival rates for each duration of the SW separately. Further implementation methods are required to find all $\gamma$-ray events that fall into the search window of a radio GP with acceptable performance. First of all, all events are loaded into memory, checked against the time windows of overlapping multi-wavelength coverage and stored in two lists for radio and $\gamma$-ray BATs, respectively. Both lists are sorted afterward in time. The analysis script then loops over all radio GPs in the first list. The monotonously increasing BAT of the radio GPs and the sorted nature of the $\gamma$-ray BAT list allow two important optimization steps: (1) Every $\gamma$-ray event that happened before the start of the "before" window with the longest duration relative to the last radio event, comes too early in time to fall into any search window relative to the current radio GP. (2) Once one $\gamma$-ray event falls past the end of the "after" search window of the longest duration, no subsequent $\gamma$-ray event will fall into any search window relative to the current radio GP. This procedure reduced the number of $\gamma$-ray events which need to be considered significantly. The calculation of the time difference between the $\gamma$-ray event and the radio GP and the conversion into pulsar periods can be skipped entirely for a large fraction of $\gamma$-ray events. The computation is not particularly time-consuming, but the large number of combinations between every radio event and every $\gamma$-ray event would produce a large overall run time. The first optimization requires that a marker is maintained for the last $\gamma$-ray event which was too early for the previous radio GP. When the iteration for the current radio GP starts at that point in the list of $\gamma$-ray BATs and an event is found which is before the start of the first search window, the marker needs to be incremented accordingly. This state is very easy and cheap to maintain and incurs no noticeable performance cost. 